\title{
SIMULATING POWDER BED BASED ADDITIVE MANUFACTURING PROCESSES: FROM DEM CALIBRATION TO EXPERIMENTAL VALIDATION
}

\author{
N. Preud'homme ${ }^{1}$, A. Neveu ${ }^{2}$, F. Francqui ${ }^{2}$, E. Opsomer ${ }^{1}$, N. Vandewalle $^{1}$ and G. \\ Lumay $^{1}$ \\ ${ }^{1}$ GRASP laboratory, CESAM research center, University of Liège, Belgium \\ ${ }^{2}$ GranuTools, Awans, Belgium
}

Key words: Additive manufacturing, DEM, powder, powder characterization, powder layer

\begin{abstract}
Powder flowability is a critical parameter for additive manufacturing techniques involving powders. In order to obtain thin and homogenous powder layers, a compromise between grain size and flowability has to be found. Unfortunately, when the grain size decreases, the cohesiveness increases and the flowability decreases. Too often, both the powder spreadability assessment and the optimization of printing parameters are costly empiric processes. In this paper, we describe an original method associating GranuDrum powder flow characterization instrument and DEM numerical simulations to asses the process-ability of powders and to optimize printing parameters like recoater speed, layer thickness or recoater geometry. The powder characterization allows to calibrate the simulation parameters and in particular to quantify the inter-grain cohesiveness. Then, the recoating process is simulated with the calibrated simulations to predict the behaviour of the powder inside the printer. In parallel, the results are validated by testing the powder in a printer equipped with an in-situ powder layer homogeneity tester based on image analysis.
\end{abstract}

\section{Introduction}

In SLS (selective laser sintering), SLM (selective laser melting), and EBM (electron beam melting) 3D printing techniques, successive thin layers of powder are created and partially sintered or melted with an energy (laser or electron) beam [1]. The vertical resolution is defined by the layer thickness and a thin layer leads to a better resolution. In order to obtain a thin layer, the powder should be as fine as possible. Unfortunately, when the grain size decreases, the cohesiveness increases and the flowability decreases $[2,3,4]$. Therefore, a compromise between grain size and flowability has to be found. The powder flowability must be good enough to obtain homogenous successive layers.

Testing a powder directly in the printer is costly and time consuming. Therefore, the powder flowability should be characterised previously in laboratory. Unfortunatelly, powder and granular materials have complex behavior and many fundamental questions are still open $[5,6,7]$. Different recent publications are evidencing that many classical flowmeters are unable to give pertinent information about powder flow behavior in powder-bed-based additive manufacturing [8]. The measurement method should be as close as possible to the process. In particular, the stress state and the flow field of the powder should 
be comparable. We have shown recently that the measurement method based on the rotating drum is a good candidate because the powder flow is analyzed precisely at the powder/air interface without any compressive load [9].

Once the powder spreadability has been assessed with the rotating drum measurement the printing parameters (for exemple recoater shape/material and speed) could be optimized. To perform this optimization task, DEM simulation is an interesting tool. However, the simulation must be calibrated to reproduce accuratelly the bahaviour of the considered powder during the simulated printing process.

In this paper, we show how the rotating drum measurement performed with GranuDrum instrument allows to predict the spreadability of a powder inside a 3D printer. In addition, the GranuDrum results are used to calibrate a DEM model to simulate the recoating process. In particular, results obtained with 2D simulations are shown as a proof of concept. The perspective is to perform 3D simulation of the exact printer geometry to investigate the effect of the different printing parameters.

\section{Powder flow characterization}

The GranuDrum instrument is an automated powder flowability measurement technique based on the rotating drum principle [10]. A horizontal cylinder with vertical glass sidewalls called drum is half filled with the sample of powder. The drum rotates around its axis at an angular velocity ranging from 2 RPM to 60 RPM for the present study. A CCD camera takes snapshots (50 images separated by $0.5 \mathrm{~s}$ ) at each angular velocity. The air/powder interface is detected on each snapshot with an edge detection algorithm. Afterward, the average interface position and the fluctuations around this average position

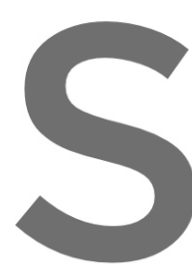
are computed. Then, for each rotating speed, the
computed from the average interface position and
interface fluctuations. Indeed, interface fluctuations
grains. This dynamic cohesive index $\sigma$ isclose to z
cohesive forces intensify.
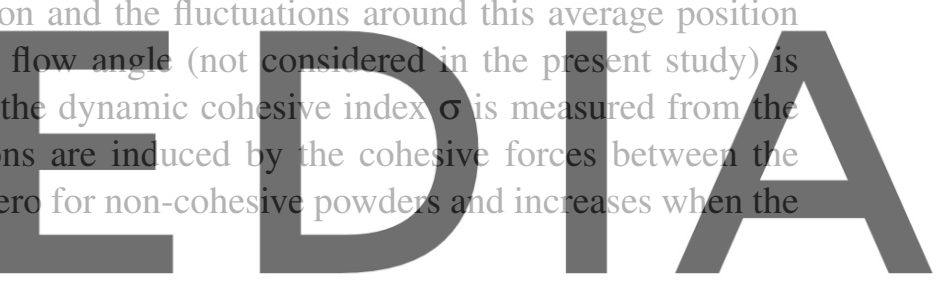

Two powders were considered: a non-cohesive powder (AlSi7Mg06 with $d_{10}=20 \mu \mathrm{m}$ and $d_{90}=63 \mu \mathrm{m}$ )

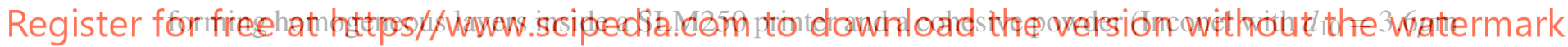
and $d_{90}=22 \mu \mathrm{m}$ ) showing a bad spreadability in the printer. Figure 1 shows typical pictures of the flow inside the rotating drum for both powders and also pictures of the layers taken inside the printer. With the Inconel cohesive powder, the powder bed shown waves and irregularities altering drastically the quality of the part produced during the printing process.

Figure 2 shows the cohesive index measured with GranuDrum at different rotating speeds. On the whole range of speeds, the Inconel cohesive powder show a higher cohesive index. Hereafter, we will use this cohesive index to calibrate the simulation parameters, in particular to calibrate the granular Bond number Bo.

\section{DEM simulations}

In order to reproduce the granular flow inside the rotating drum, a in-house algorithm based on the softparticle Discrete Element Method (DEM) has been used [11, 12]. The forces acting on each individual grains are calculated at a chosen discrete time step as well as their updated velocity. The normal contact forces are modeled using a linear spring dashpot and the tangential forces are computed according to Coulomb's law of friction which is modeled with a linear spring for static friction. 


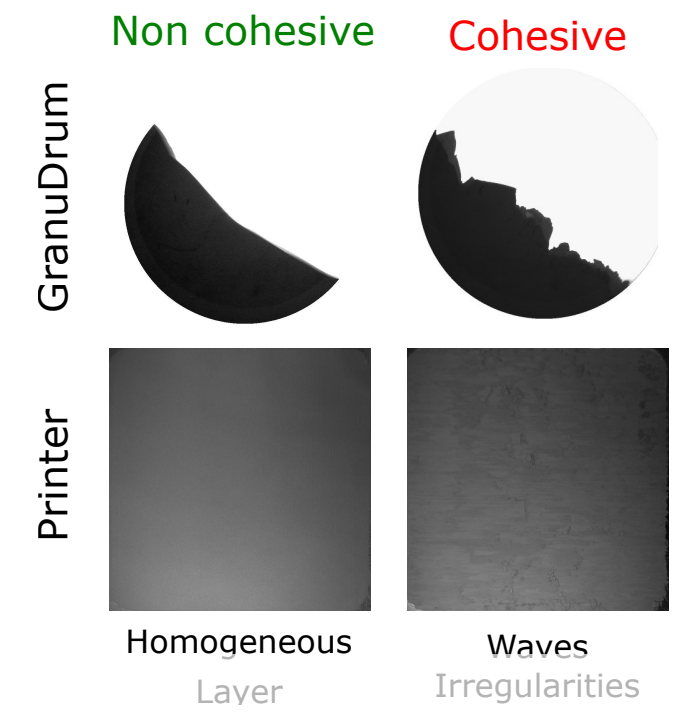

Figure 1: (Top) Typical pictures of the flow inside the rotating drum for a non-cohesive powder and a cohesive powder. (Bottom) Picture on the powder layer obtained inside the 3D printer with the same powders.
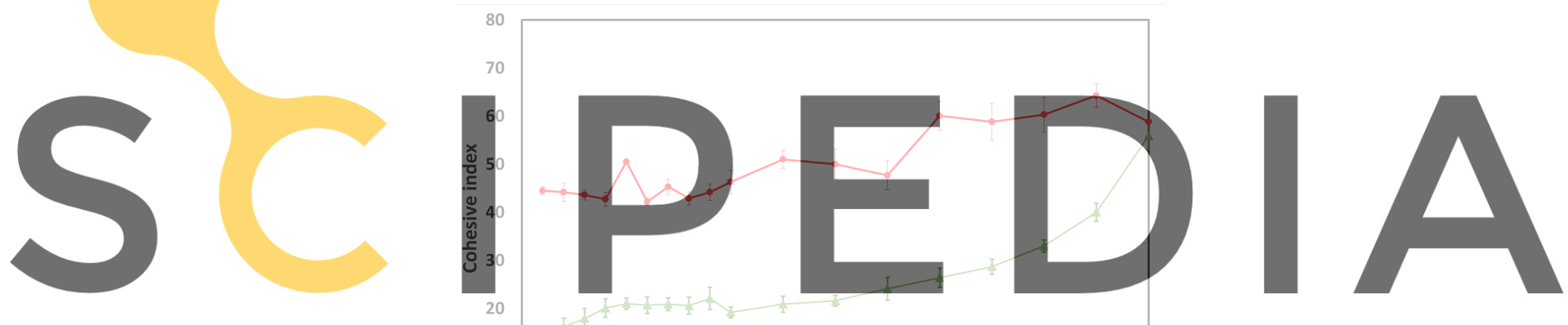

Register for free at https//ww'hl.scipedia.com to to download the version without the watermark

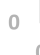

20

Rotating speed (rpm)

Figure 2: Cohesive index measured with GranuDrum as a function of the drum rotating speed, i.e. for different flowing speed. The cohesive index is measured from the fluctuations of the grainular/air interface.

A simple expression for the cohesive force has been voluntarily chosen to mimic a large panel of the different physical cohesive interactions which are encountered. The model considers maximum attraction at contact which decreases quadratically with the grain inter-distance $\delta$. Curvature is chosen so that the attractive force vanishes at a fixed range corresponding to the radius $r$ of a grain i.e. $\delta= \pm r$. The intensity of this attraction force is expressed with the Bond number $B o$. This dimensionless number is defined as the ratio between the attractive force to the weight of the grains.

$$
B o=\frac{F_{c}}{m g}
$$


To fulfill these conditions, we defined the attractive force between grains as follows

$$
\mathbf{F}_{\mathbf{c}}=m g B o\left(\left(\frac{\delta}{r}\right)^{2}-1\right) \hat{\mathbf{n}}
$$

with $r$ the grain radius and $\hat{\mathbf{n}}$ the unitary vector pointing from the center of one grain to the other. These forces are also valid for contacts between grains and the walls forming the system except for cohesion which is not implemented for this type of interaction involving grains and the walls.

In the framework of the present study aiming to show the methodology to combine experiments et simulations, we focused on 2D simulations for the flow in the rotating drum and also for the simulation of the recoating process with a simple geometry represented in Figure 3 . To reproduce the additive manufacturing process in 2D, a rectangular container with a length $L \sim 300$ grain diameters and a height $h \sim 15$ grain diameters is first filled up with grains. The grains have a coefficient of restitution of 0.2 , a friction coefficient of 0.9 and their size distribution is slightly polydisperse as their radius is given by $r \pm 7.5 \%$. The successive layers of grains are deposited on the powder bed by a recoater simply consisting of 2 vertical walls, separated by $1 / 10^{\text {th }}$ of the container length, sweeping the surface of the powder bed and between which grains are dropped after each back and forth passage over the container. The speed of the recoater is set to $1 / 4^{\text {th }}$ container length per second. The height of the container is increased by 3 grain diameters after each passage of the recoater by slowly moving the bottom horizontal wall forming the container. This way, a 3-grain-diameter thick new layer of grains can be deposited on the underlying powder bed. With Cohesive powders, fluctuations of the granular/air interface are observed in the drum

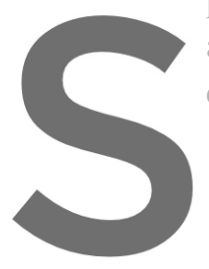
and also inside the simulated pri
comparable to the experimental
(a)
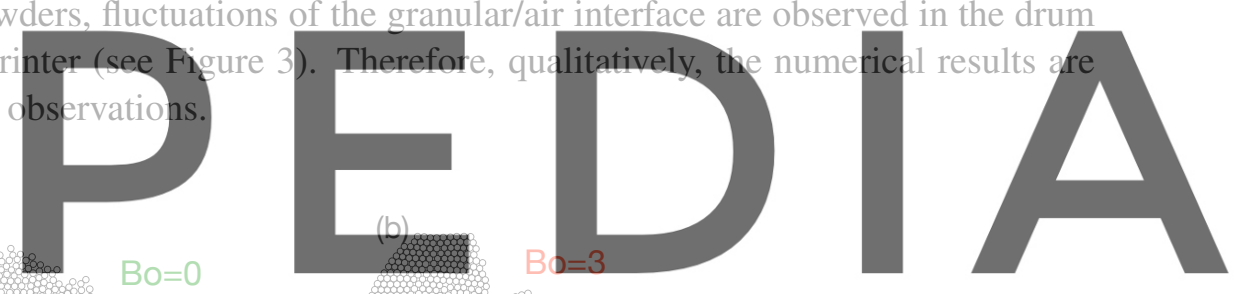

Register for free at https//www.scipedia.com to download the version without the watermark

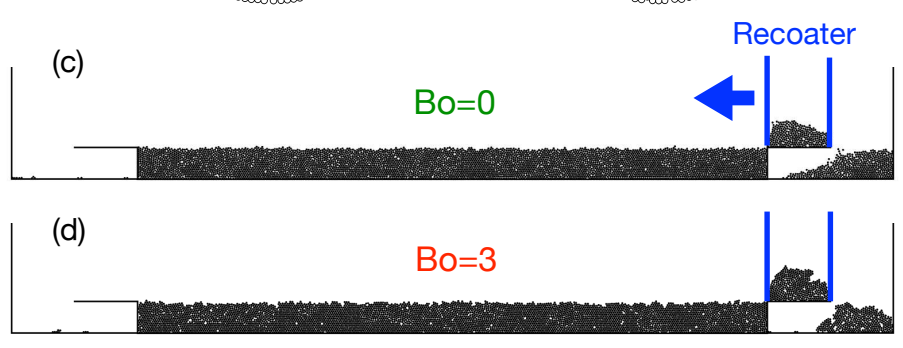

Figure 3: $(\mathrm{a}, \mathrm{b})$ Typical pictures of the flow inside the simulated rotating drum for a non-cohesive powder $B o=0$ (a) and a cohesive powder $B o=3$ (b). (c,d) Pictures of layer formation obtained with DEM simulation also for a non-cohesive powder $B o=0$ (c) and a cohesive powder $B o=3$ (d).

To connect quantitatively simulations and experiments, the granular Bond number $B o$ is used. A set 
of simulations was performed with the rotating drum at the intermediate rotating speed of 30RPM for different values of the Bond number Bo. Afterward, the algorithm of GranuDrum instrument was used to measure the cohesive index from a set of 50 images taken from each simulation (see Figure 4). By comparing experimental (Figure 2) and numerical (Figure 4) results, the Bond numbers corresponding to the powders can be estimated. Beyond the framework of the present study, the quantification of the cohesive forces inside a powder could be useful for many applications.

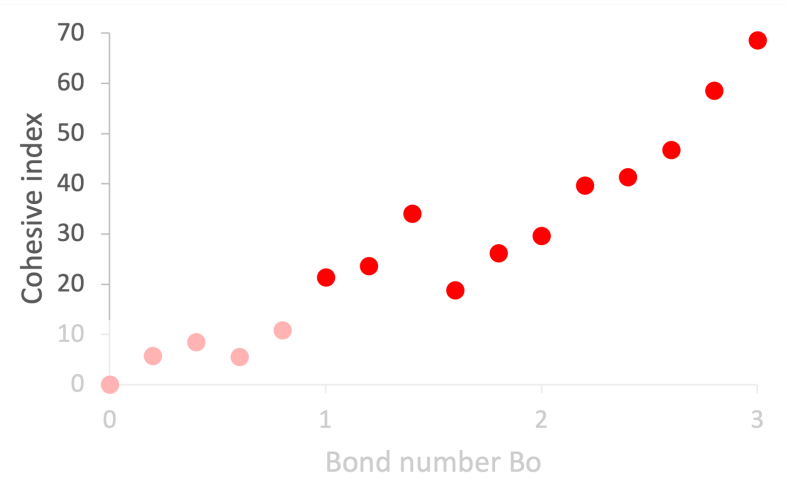

Figure 4: Cohesive index obtained from the interface fluctuations in the simulated rotating drum as a function of the Bond number Bo characterizing the cohesiveness. This plot allows callibrate the simulation, i.e. to select the Bond number corresponding to the powder.
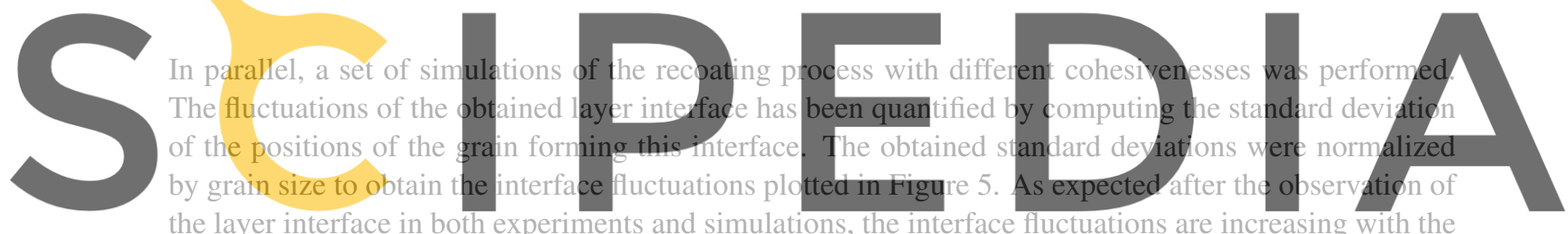

the layer interface in both experiments and simulations, the interface fluctuations are increasing with the

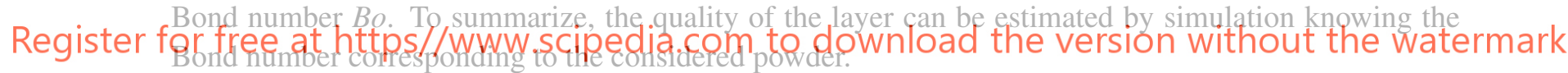

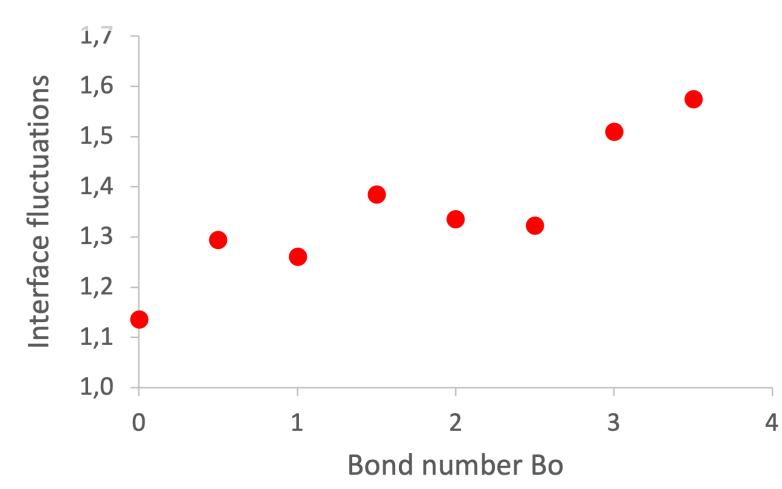

Figure 5: Layer fluctuations (standard deviation of the positions of the grains forming the interface) in the simulated printing process as a function of the Bond number $B o$. 


\section{Conclusion}

We showed a methodology to estimate the quality of the powder layers inside a 3D printer using numerical simulations combined with measurements. First, the powders is characterised using GranuDrum instrument. More precisely, the cohesive index of the powder is measured. Afterward, the cohesive index is used to calibrate the simulation. In particular, the Bond number corresponding to the cohesive index is estimated by simulating the rotating drum. Finally, the recoating process is simulated to estimate the quality of the layers. As a perspective, this methodology could allow the investigation of the effect of printing parameters like the recoating speed or the recoater geometry.

\section{REFERENCES}

[1] Olakanmi E.O., Cochrane R.F. and Dalgarno K.W. A review on selective laser sintering/melting (SLS/SLM) of aluminium alloy powders: Processing, microstructure, and properties. Progress in Materials Science (2015) 74:401-477.

[2] Ruggi D., Barres C., Charmeau J.-Y., Fulchiron R., Barletta D., Poletto M. A quantitative approach to assess high temperature flow properties of a PA 12 powder for laser sintering. Additive Manufacturing (2020) 33:101143.

[3] Ligon S. C., Liska R., Stampfl J., Gurr M., Mulhaupt R. Polymers for 3D Printing and Customized Additive Manufacturing, Chem. Rev. (2017) 117:10212-10290.

[4] Castellanos A., Perez A.T., Ramos A., Watson P.K. Flow regimes in fine cohesive powders, Phys.
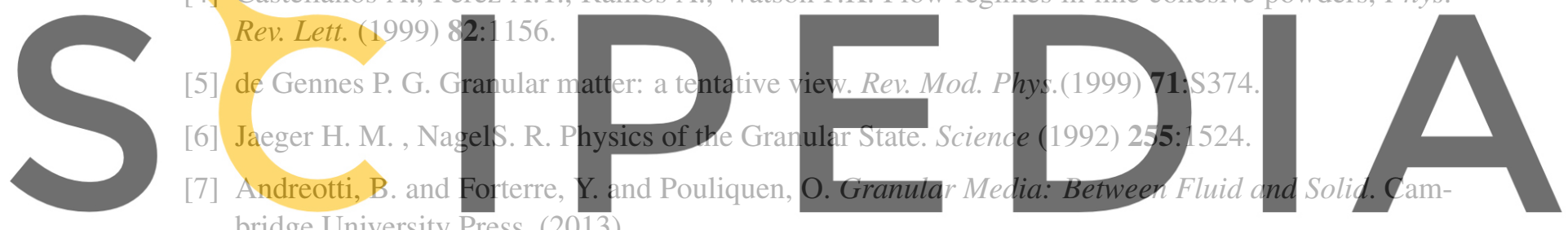
bridge University Press, (2013)

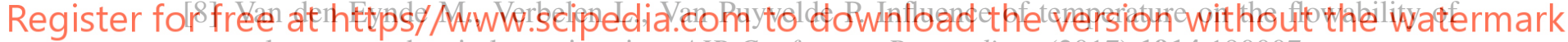
polymer powders in laser sintering. AIP Conference Proceedings (2017) 1914:190007.

[9] Yablokova G., Speirs M., Van Humbeeck J., Kruth J.-P., Schrooten J., Cloots R., Boschini F., Lumay G., and Luyten J. Rheological behavior of $\beta$-Ti and NiTi powders produced by atomization for SLM production of open porous orthopedic implants. Powder Technology (2015) 283:199.

[10] G. Lumay, F. Boschini, K. Traina, S. Bontempi, J.-C. Remy, R. Cloots, N. Vandewall, Measuring the flowing properties of powders and grains, Powder Technology (2012) 224:19.

[11] Luding, S. Cohesive, frictional powders: Contact models for tension. Granular Matter (2008) 10:235-246.

[12] Opsomer E., Ludewig F., Vandewalle N. Phase transitions in vibrated granular systems in microgravity. Physical Review E (2011) 84:051306 